\title{
Diversity Improves Teamwork: Optimising Teams using a Genetic Algorithm
}

\author{
Soo Ling Lim \\ Braintree Research Lab, Braintree Ltd \\ Department of Computer Science, UCL \\ London, United Kingdom \\ s.lim@cs.ucl.ac.uk
}

\author{
Peter J. Bentley \\ Braintree Research Lab, Braintree Ltd \\ Department of Computer Science, UCL \\ London, United Kingdom \\ p.bentley@cs.ucl.ac.uk
}

\begin{abstract}
Collaboration is fundamental to our society, but how should we best build teams? We investigate by applying optimisation to an agent-based model of collaboration. The model takes inspiration from particle swarm optimisation, abstracting a shared goal as a shared optimisation task, and modelling the personality differences in team members as strategies for moving within, interpreting and sharing information about the solution space. We update the model and add a genetic algorithm in order to investigate the effects of differing initial ideas within teams of different personality combinations as they attempt to collaborate to achieve their shared task. We run experiments on homogeneous teams with similar personalities and heterogeneous teams with random personalities and find that increased diversity in team members' initial ideas significantly improves teamwork, and more so for teams comprising individuals with similar personalities.
\end{abstract}

Keywords-agent-based modelling, genetic algorithm, collaboration, teamwork, personality psychology

\section{INTRODUCTION}

Collaboration forms the cornerstone of human society. By working together, we achieve extraordinary feats of science, engineering, agriculture, medicine - indeed, all our advancements throughout human history. Today there is an ever-greater need for our teamworking to be effective, and increasing attention is being placed on finding the right combination of people to work together for a given task. Every individual is unique and is shaped by their background - so how to choose the right people for a team? Should we pick individuals who share similar initial ideas about the task, to minimise conflict? (This might be reflected by having similar places of education, similar socioeconomic background, or similar culture.) Should we pick individuals who all have similar personalities, because perhaps they will work together more effectively? Or conversely should we choose individuals who have completely different backgrounds, and start the task with radically differing ideas? Should we pick individuals with contrasting personalities, so that they might discuss more and generate original ideas? This work investigates these questions through the use of a genetic algorithm applied to a computational model of human teamwork.

To study teamwork in this way we consider participants who have shared goals, but may have varied decision-making strategies involving different methods of communication and data processing. This can be contrasted with the frequentlystudied notion of cooperation, where it is assumed that participants have separate goals and all make decisions using the same information. For example, a prisoner may make a rational choice to cooperate with a police officer despite having a different goal. In contrast, two police officers collaborate together because they have shared goals, but they may do so ineffectively if their backgrounds differ or decision-making strategies (personalities) conflict [1].

To understand how best to optimise human teams, we study collaboration, and in order to model different decision-making strategies, we use an agent-based model of collaboration, taking inspiration from particle swarm optimisation, that abstracts a shared goal as a shared optimisation task, and models the personality differences in team members as strategies for moving within, interpreting and sharing information about the solution space. In previous work we used the model to explore how teams with different personalities work together when the shared optimisation task has different levels of noise [1]. In this work, we modify the model in order to improve its speed, and add optimisation in the form of a genetic algorithm (GA) in order to investigate the effects of differing initial ideas within teams of different personality combinations as they attempt to collaborate to achieve their shared task.

This paper is organised as follows. Section II describes related work. Section III describes the model, Section IV describes the genetic algorithm used to find the best and worst starting positions of teams, Section $\mathrm{V}$ describes the experiments, Section VI discusses the results and Section VIII concludes.

\section{BACKGROUND}

The use of agent-based modelling for human behaviour focussing on the effects of personalities has been performed by a handful of researchers so far. GAs have also been used to optimise agent-based models in general. The following two sections describe some examples of such work. To date there has been no work that applies a GA to an agent-based model of human teamwork that incorporates personalities in order to optimise teams based on their backgrounds. 


\section{A. Agent-based Models of Personality in Collaboration}

Salvit and Sklar [2] used the Myers-Briggs Type Indicator (MBTI) to model termites gathering food. The MBTI is a widely used personality inventory based the theory of psychological types by Jung [3] that classifies individuals based on their attitude (Extraverted vs. Introverted), perception (Sensing vs. Intuition) and judgment (Thinking vs. Feeling). In the termite model, Sensing agents focus on food that is close by and return to the place they last saw food if they cannot see food, while Intuitive agents prefer bigger clusters of food and explore new areas when they cannot see food. Thinking agents go straight for their targeted food, while Feeling agents avoid food that their neighbours are targeting.

Campos et al. [4] simulated a firefighting scenario where a building is on fire and a person is in danger. Firefighter agents with different MBTI personality types can either put a safety-net in place for the person to jump on it or enter the building to bring the person out. They found that Sensing agents prefer to use the safety-net and Intuitive agents prefer to enter the building.

Ahrndt et al. [5] used the five-factor model (FFM) to model ants in a colony working together to collect food and defend themselves. The FFM consists of five personality traits: openness to experience, conscientiousness, extraversion, agreeableness and neuroticism, and is widely used in personality research [6]. In the ant colony model, agreeableness and extraversion influence an agent's preference to commit to selfish or altruistic goals, and variations on conscientiousness influence an agent's preference to change their intentions.

Durupinar [7] extended the High-Density Autonomous Crowds system using the FFM. They provided each agent with personalities that are associated with an existing behaviour in the system and found that specifying an agent's personality leads to an automation of low-level parameter tuning. In their model, people with low conscientiousness and agreeableness cause congestion and neurotic people display panic behaviour.

\section{B. Optimising Agent-based Models with Genetic Algorithms}

Knoester et al. [8] used the AVIDA platform [9] to study the evolution of consensus, a cooperative behaviour in which members agree on information sensed in their environment. They used a form of evolutionary computation where a population of digital organisms is subject to instruction-level mutations and natural selection and placed them into groups whose fitness relied upon their ability to perform consensus. They then tested different degrees and types of genetic variation present in the population, based on biologically inspired models of gene flow. Their experiments found that while genetic heterogeneity within groups increases the difficulty of the consensus task, a surprising number of groups were able to overcome these obstacles and evolve this cooperative behaviour.

Heppenstall et al. [10] used a genetic algorithm to optimise an agent-based model for simulating a retail market. They model individual petrol stations as agents and integrated important additional system behaviour through the use of established methodologies such as spatial interaction model. The parameters were initially determined by the use of real data analysis and experimentation and then a GA is used to produce not just an optimised match, but results that match those derived by expert analysis through rational exploration.

Wang et al. [11] used a genetic algorithm to optimise partner selection for virtual enterprises. They develop a genetic algorithm that finds the partners that reduce their collaboration time and cost. They consider three types of collaborations: logistics, information transmission and capital flow and two task allocation scenarios: allocating all tasks to one partner and allocation each task to different partners.

\section{The Personality Agent-Based Model}

In our previous work [1], we proposed an agent-based model of human collaboration inspired by particle swarm optimisation to simulate the differing behaviours of people according to their personalities. Our model has the following key abstractions:

- Problem. We abstract the shared goal of all agents as the shared task to optimise a function (i.e., find the values of $\mathbf{x}$ such that $f(\mathbf{x})$ is maximised).

- Agent psychology. Inspired by swarming algorithms, we model the current mental state of each agent by giving it a position in the solution space (denoting the solution its mind has found so far), a velocity vector (denoting the direction and speed of its thought process), and acceleration vectors (representing the force of ideas and influences that modify the direction and speed of thought), the latter determined by its personality.

- Agent communication. We model the distribution of information between agents as they each try to solve the same problem. The exact type of information perceived by each agent and its use is determined by its personality.

Fig. 1 shows the algorithm of the model, and the following sections describe each component in detail.

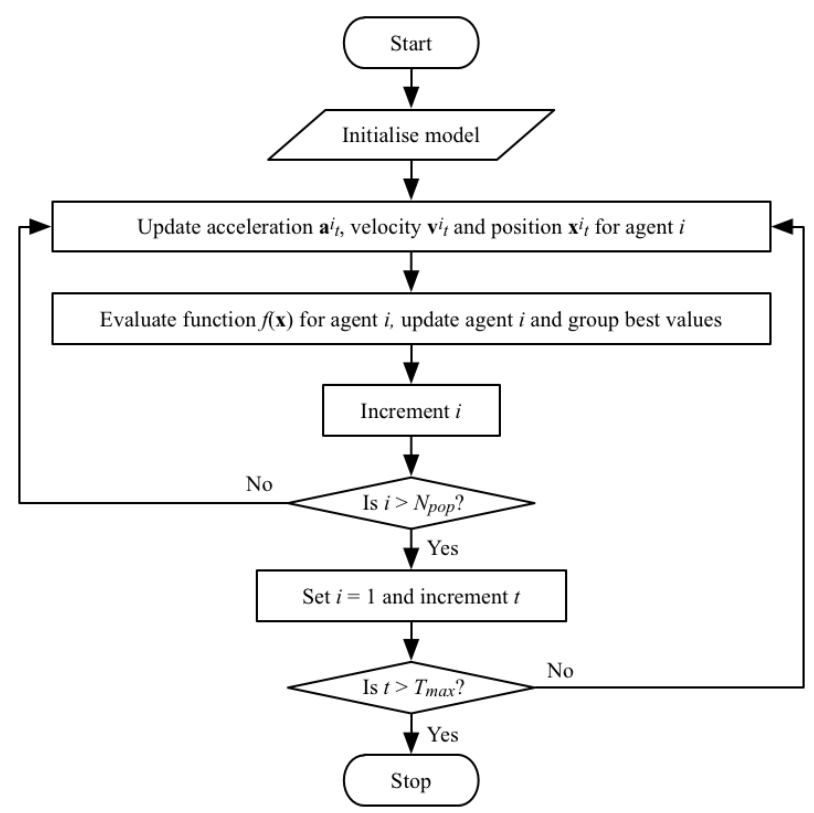

Fig. 1. Algorithm of the model [12]. 


\section{A. Initialise}

The model is initialised with:

- a problem space $\mathbf{D} \in \mathbb{R}^{n}$

- an objective function $f(\mathbf{x})$

- the number of timesteps $T_{\text {max }}$ to run the model

- a population of agents $N_{\text {pop }}$, each agent $i \in\left\{1, \ldots, N_{\text {pop }}\right\}$ is initialised with:

$\circ$ a personality type $\mathbf{P}^{i}$ (one of the MBTI personality types described in the next section)

$\circ$ a position $\mathbf{x}_{0}^{i} \in \mathbf{D}: \mathbf{x}_{\min } \leq \mathbf{x}^{i}{ }_{0} \leq \mathbf{x}_{\max }$, derived from a corresponding genotype (see Section IV)

$\circ$ a random velocity $\mathbf{v}_{0}{ }_{0} \in \mathbb{R}^{n}:-\mathbf{v}_{\text {init }} \leq \mathbf{v}^{i}{ }_{0} \leq \mathbf{v}_{\text {init }}$

$\circ$ personal best $f_{\text {best }}^{i}=f\left(\mathbf{x}_{0}^{i}\right)$ and personal best position $\mathbf{x}_{\text {best }}^{i}=\mathbf{x}^{i}{ }_{0}$

- group best $f^{g}{ }_{\text {best }}$ is the best $f^{i}$ best, and group best position $\mathbf{x}^{g}{ }_{\text {best }}$ is the corresponding $\mathbf{x}^{i}$ best

\section{B. Update}

For each timestep $t \in\left\{1, \ldots, T_{\max }\right\}$, each agent $i$ 's position $\mathbf{x}^{i}{ }_{t}$ is updated using Equation 1:

$$
\mathrm{x}_{\mathrm{t}}^{\mathrm{i}}=\mathrm{x}_{\mathrm{t}-1}^{\mathrm{i}}+\mathrm{v}_{\mathrm{t}}^{\mathrm{i}}
$$

with the velocity $\mathbf{v}^{i}{ }_{t}$ calculated using Equation 2:

$$
\mathrm{v}_{\mathrm{t}}^{\mathrm{i}}=\mathrm{v}_{\mathrm{t}-1}^{\mathrm{i}}+\mathrm{a}_{\mathrm{t}}^{\mathrm{i}}
$$

If $\left|\mathbf{v}_{t}{ }_{t}\right|>\mathbf{v}_{\text {max }}$, it is scaled to equal $\mathbf{v}_{\text {max }}$, in order to prevent excessive speed (an individual with high velocity would literally become too "set in their ways" and would find it impossible to change its direction of thought into a useful direction).

Acceleration $\mathbf{a}^{i}{ }_{t}$ is used to change the direction and speed of thought, as determined by the agent's personality - one of the 16 MBTI personality types [13]. The interpretation provided here is designed to enable each personality to have an equally good chance of finding the solution. Interpretations were created to represent MBTI personality types appropriately and were not tuned in order to achieve any specific result in later experiments.

The MBTI consists of 16 personality types based on a person's preferences on four opposing dichotomies: Extraversion (E) - Introversion (I), Sensing (S) - Intuition (N), Thinking (T) - Feeling (F), and Judging (J) - Perceiving (P) [13]. J - P defines the person's preferred manner (either S-N or T-F) of dealing with the outer world. Each personality type has a dominant Jungian function (more developed) supported by an auxiliary Jungian function (less developed) as shown in Table I.

Table II defines the Jungian attitude, and perception and judgment functions. Each attitude (extraversion and introversion) is used as a source of information for each function (Thinking, Feeling, Sensing, Intuition), resulting in Jung's eight psychological types: extraverted Thinking (Te), introverted Thinking (Ti), extraverted Feeling (Fe), introverted Feeling ( $\mathrm{Fi}$ ), extraverted Sensing (Se), introverted Sensing (Si), extraverted iNtuition $(\mathrm{Ne})$, introverted iNtuition $(\mathrm{Ni})$.

Acceleration $\mathbf{a}^{i}{ }_{t}$ is calculated using Equation 3:

$$
\mathrm{a}_{\mathrm{t}}^{\mathrm{i}}=\mathrm{a}_{\mathrm{J}}{ }_{\mathrm{t}}^{\mathrm{i}}+\mathrm{a}_{\mathrm{P}_{\mathrm{t}}}^{\mathrm{i}}
$$

where $\mathbf{a}_{J}{ }_{t}{ }_{t}$ is the judging acceleration is calculated using Table III and $\mathbf{a}_{P}{ }^{i}{ }_{t}$ is the perceiving acceleration calculated using Equation 4:

$$
\mathrm{a}_{\mathrm{P}^{\mathrm{i}}}{ }_{\mathrm{t}}=\sum_{\mathrm{j}=1}^{3} \mathrm{r}_{\mathrm{j}}\left(\mathrm{c}_{\mathrm{j}}-\mathrm{x}^{\mathrm{i}}{ }_{\mathrm{t}-1}\right)
$$

where $r_{1}=0.5, r_{2}=0.3$, and $r_{3}=0.2$, and $\mathbf{c}_{1}, \mathbf{c}_{2}$ and $\mathbf{c}_{3}$ are the top 3 candidates derived using Table IV with $f\left(\mathbf{c}_{1}\right) \geq$ $f\left(\mathbf{c}_{2}\right) \geq f\left(\mathbf{c}_{3}\right)$. In both tables, agent $i$ 's neighbours are defined as the five nearest agents to agent $i$ measured by Euclidean distance, i.e., the peer group of each agent comprises those who share similar ideas to the agent. To ensure that the auxiliary component plays a lesser role compared to the dominant component, $\mathbf{a}_{J}{ }_{t}{ }_{t}$ is scaled down such that $\left|\mathbf{a}_{J}{ }_{t}\right|^{2}=\frac{\mid \mathbf{a}_{P}{ }^{i} t^{2}}{2}$ if $\left|\mathbf{a}_{J}{ }_{t}{ }_{t}\right|^{2}>\frac{\left|\mathbf{a}_{P}{ }_{t}\right|^{2}}{2}$ (if $\mathbf{P}^{i}$ has dominant perception and auxiliary judgment, otherwise vice versa).

\section{Evaluate}

Agent $i$ 's fitness at timestep $t$ is evaluated as $f^{i}{ }_{t}=f\left(\mathbf{x}^{i}{ }_{t}\right)$. Finally, the agent's personal best $f^{i}{ }_{\text {best }}$, the agent's personal best position $\mathbf{x}_{\text {best }}^{i}$, group best $f^{g}{ }_{\text {best }}$ and group best position $\mathbf{x}^{g}{ }_{\text {best }}$ are updated.

TABle I. Myers-Briggs Type TABle Showing the 16 Personality Types, WITH DOMINANT AND AUXILIARY FUNCTIONS [13]

\begin{tabular}{|c|c|c|c|c|}
\hline Type & ISTJ & ISFJ & INFJ & INTJ \\
Dominant & $\mathrm{Si}$ & $\mathrm{Si}$ & $\mathrm{Ni}$ & $\mathrm{Ni}$ \\
Auxiliary & $\mathrm{Te}$ & $\mathrm{Fe}$ & $\mathrm{Fe}$ & $\mathrm{Te}$ \\
\hline Type & ISTP & ISFP & INFP & INTP \\
Dominant & $\mathrm{Ti}$ & $\mathrm{Fi}$ & $\mathrm{Fi}$ & $\mathrm{Ti}$ \\
Auxiliary & $\mathrm{Se}$ & $\mathrm{Se}$ & $\mathrm{Ne}$ & $\mathrm{Ne}$ \\
\hline Type & ESTP & ESFP & ENFP & ENTP \\
Dominant & $\mathrm{Se}$ & $\mathrm{Se}$ & $\mathrm{Ne}$ & $\mathrm{Ne}$ \\
Auxiliary & $\mathrm{Ti}$ & $\mathrm{Fi}$ & $\mathrm{Fi}$ & $\mathrm{Ti}$ \\
\hline Type & ESTJ & ESFJ & ENFJ & ENTJ \\
Dominant & $\mathrm{Te}$ & $\mathrm{Fe}$ & $\mathrm{Fe}$ & $\mathrm{Te}$ \\
Auxiliary & $\mathrm{Si}$ & $\mathrm{Si}$ & $\mathrm{Ni}$ & $\mathrm{Ni}$ \\
\hline
\end{tabular}

TABle II. Jungian Attitudes, Perceptions, And Judgments [3]

\begin{tabular}{|l|l|}
\hline Attitude & $\begin{array}{l}\text { Extraversion: Directs perception and judgment on outer world } \\
\text { of people and things. }\end{array}$ \\
\cline { 2 - 3 } & $\begin{array}{l}\text { Introversion: Directs perception and judgment on outer world } \\
\text { of people and things. }\end{array}$ \\
\hline Perception & $\begin{array}{l}\text { Sensing: Concrete perception, finds interest in actualities (made } \\
\text { aware directly through the senses), prefers not to go beyond the } \\
\text { objective, empirical world of facts. Relies on concrete, actual } \\
\text { information. }\end{array}$ \\
\cline { 2 - 3 } & $\begin{array}{l}\text { Intuition: Abstract perception, finds interest in connecting } \\
\text { concepts and drawing parallels (made aware indirectly by way } \\
\text { of the unconscious). Relies upon their conception about things } \\
\text { based on their own understanding. }\end{array}$ \\
\hline Judgment & $\begin{array}{l}\text { Thinking: Impersonal assessment, comes to conclusions based } \\
\text { on a logical process, aimed at an impersonal finding (facts and } \\
\text { ideas), analyses and determines the truth or falseness of } \\
\text { information in an impersonal fashion. }\end{array}$ \\
\cline { 2 - 3 } & $\begin{array}{l}\text { Feeling: Person-centred assessment, comes to conclusions } \\
\text { based on a process of appreciation, giving things a personal, } \\
\text { subjective value. }\end{array}$ \\
\hline
\end{tabular}




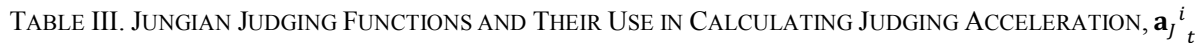

\begin{tabular}{|c|c|}
\hline Function & Implementation \\
\hline $\begin{array}{l}\text { Te: The agent is influenced by its neighbours' best personal } \\
\text { best. It accelerates towards its neighbours' best personal } \\
\text { best from the previous timestep. }\end{array}$ & $\begin{array}{l}\mathbf{a}_{T e^{i}{ }_{t}}=\mathbf{x}_{n}{ }^{i}{ }_{\text {best }}-\mathbf{x}_{t-1}{ }_{t-1} \\
\text { where } \mathbf{x}_{n}{ }^{i}{ }_{\text {best }}{ }_{t-1} \text { is agent } i \text { 's neighbours' personal best position in the previous timestep that } \\
\text { results in the highest } f(\mathbf{x}) \text {, and } \mathbf{x}^{i}{ }_{t-1} \text { is the agent's position in the previous timestep. }\end{array}$ \\
\hline $\begin{array}{l}\text { Ti: The agent focusses on its own personal best (the } \\
\text { outcome of its own thoughts). It accelerates towards its } \\
\text { own personal best, with randomness added to enable } \\
\text { exploration. }\end{array}$ & 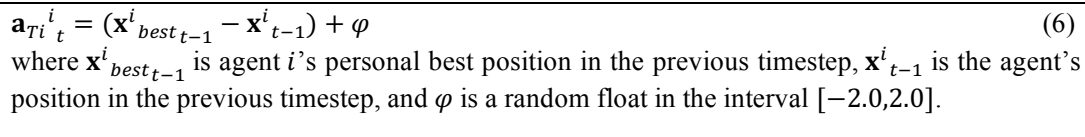 \\
\hline $\begin{array}{l}\text { Fe: The agent "identifies with other agent's feelings" and } \\
\text { "seeks harmony" by matching its neighbours' average } \\
\text { velocity (direction of thought) from the previous timestep } \\
\text { and to a lesser extent accelerates towards its neighbours" } \\
\text { best personal best from the previous timestep. }\end{array}$ & $\begin{array}{l}\mathbf{a}_{F e}{ }_{t}^{i}=\omega_{1} \cdot \overline{\mathbf{v}}_{N}{ }^{i}{ }_{t-1}+\omega_{2} \cdot \mathbf{a}_{T e}{ }^{i}{ }_{t} \\
\text { where weights } \omega_{1}=0.8, \omega_{2}=0.2, \overline{\mathbf{v}}_{N}{ }^{i}{ }_{t-1} \text { is agent } i \text { 's neighbours' average velocity in the } \\
\text { previous timestep, and } \mathbf{a}_{T e}{ }^{i}{ }_{t} \text { is calculated using Equation } 5 .\end{array}$ \\
\hline $\begin{array}{l}\text { Fi: The agent "empathises with" its neighbours' ideas by } \\
\text { accelerating towards its neighbours' average position from } \\
\text { the previous timestep. It also cares about its own personal } \\
\text { thoughts, so accelerates towards its own best position. }\end{array}$ & $\begin{array}{l}\mathbf{a}_{F i}{ }^{i}{ }_{t}=\omega_{1} \cdot\left(\mathbf{C}_{n}{ }^{i}{ }_{t-1}-\mathbf{x}^{i}{ }_{t-1}\right)+\omega_{2} \cdot\left(\mathbf{x}^{i}{ }_{\text {best }}+{ }_{t-1}-\mathbf{x}^{i}{ }_{t-1}\right) \\
\text { where weights } \omega_{1}=0.8, \omega_{2}=0.2, \mathbf{C}_{n}{ }^{i}{ }_{t-1} \text { is the centroid (arithmetic mean position) of agent } i \text { 's } \\
\text { neighbours' positions in the previous timestep. }\end{array}$ \\
\hline
\end{tabular}

TABle IV. Jungian Perceiving Functions AND Their Use In GetTing CANDidates. The First Three CANDidates ARE Returned AS $c_{1}, c_{2}$ AND $c_{3}$

\begin{tabular}{|c|c|}
\hline Function & Implementation \\
\hline $\begin{array}{l}\text { Se: The agent sees its neighbours' positions and their } \\
\text { quality. Candidates are the positions of the agent's nearest } \\
\text { neighbours in the previous timestep. }\end{array}$ & $\begin{array}{l}\mathbb{C}_{S e^{i}}{ }_{t}=\left\{\mathbf{x}^{i}{ }_{n 1}{ }_{t-1}, \ldots, \mathbf{x}_{n 5-1}^{i}\right\} \\
\text { where } \mathbf{x}^{i}{ }_{n 1_{t-1}} \text { is agent } i \text { 's first neighbour's position in the previous timestep, and } \mathbf{x}^{i}{ }_{n 5}{ }_{t-1} \text { is agent } \\
i \text { 's fifth neighbour's position in the previous timestep. The candidates for current and previous } \\
\text { timestep } \mathbb{C}_{S e}{ }_{t}{ }_{t} \text { and } \mathbb{C}_{S e}{ }^{i}{ }_{t-1} \text { are then sorted in the order of decreasing } f(\mathbf{x}) \text {. }\end{array}$ \\
\hline $\begin{array}{l}\text { Si: The agent remembers all its own previous positions and } \\
\text { a few nearby points and their quality. Candidates are the } \\
\text { agent's previous path and new points near to their position. }\end{array}$ & $\begin{array}{l}\mathbb{C}_{S i}{ }^{i}{ }_{t}=\left\{\mathbf{x}^{i}{ }_{0}, \ldots, \mathbf{x}^{i}{ }_{t-1}\right\} \cup \boldsymbol{P} \\
\text { where } \boldsymbol{P} \text { is the set of points near to } \mathbf{x}^{i}{ }_{t-1} \text {. Given } \mathbf{x}_{t-1}^{i}=\left(x_{1}, x_{2}, \ldots, x_{n}\right), \boldsymbol{P}=\left\{\left(x_{1}+\right.\right. \\
\left.\delta, x_{2}, \ldots, x_{n}\right),\left(x_{1}-\delta, x_{2}, \ldots, x_{n}\right),\left(x, x_{2}+\delta, \ldots, x_{n}\right),\left(x_{1}, x_{2}-\delta, \ldots, x_{n}\right), \ldots,\left(x_{1}, x_{2}, \ldots, x_{n}+\right. \\
\left.\delta),\left(x_{1}, x_{2}, \ldots, x_{n}-\delta\right)\right\} \text { where } \delta \text { is a random number from a normal distribution } N(\mu, \sigma) \text { with } \\
\mu=1 \text { and } \sigma=0.01 \text {. The candidates for current and previous timestep } \mathbb{C}_{S i}{ }^{i}{ }_{t} \text { and } \mathbb{C}_{S i}{ }^{i}{ }_{t-1} \text { are then } \\
\text { sorted in the order of decreasing } f(\mathbf{x}) \text {. }\end{array}$ \\
\hline $\begin{array}{l}\text { Ne: The agent sees its neighbours' positions and uses them } \\
\text { to create an "imaginary solution space". Candidates } \\
\text { produced from Se (data from the environment) are used as } \\
\text { input to train the Gaussian process regression function. } \\
\text { Candidates are then the best quality solutions resulting } \\
\text { from sampling this imaginary space. }\end{array}$ & $\begin{array}{l}f^{*}=\mathcal{G P}: \text { train }\left(\mathbb{C}_{S e}, f\left(\mathbb{C}_{S e}\right)\right) \text {; predict }\left(\mathbb{C}_{N e}{ }_{t}{ }_{t}\right) \\
\text { where } \mathcal{G P} \text { is the Gaussian process regression function [14], training on } \mathbb{C}_{S e} \text { and } f\left(\mathbb{C}_{S e}\right) \text {, and } \\
\mathbb{C}_{N e}{ }^{i}{ }_{t} \text { is a vector of points in } \mathbf{D} \text {. The candidates for current and previous timestep } \mathbb{C}_{N e}{ }_{t}{ }_{t} \text { and } \\
\mathbb{C}_{N e}{ }_{t-1} \text { are then sorted in the order of decreasing } f^{*} \text {. }\end{array}$ \\
\hline
\end{tabular}

\section{Updates to Model}

In this work, we updated the model by changing the $\mathbb{C}_{N e}{ }^{i}{ }_{t}$ and $\mathbb{C}_{N i}{ }^{i}{ }_{t}$ vector from all discrete points in $\mathbf{D}$ to every 10 points, in order to speed up computation. We also reduced $T_{\max }$ from 50 to 25 because fewer time steps are required to run the model when there is no noise, and we duplicate each run 50 times instead of 100 times for the same reason. We then reran previous experiments [1], verifying that our updates do not change the agent performance or trends. Finally, in [1], agents start with random positions (so people who start nearer to the solution may have some benefit - reflecting someone having a better idea of the solution at the start). Here, the distance of all agents from the solution is the same, but the distribution of agents on this circle is varied by a genetic algorithm, see Section IV.

\section{E. Settings and Function}

The model was initialised with constant settings in Table $\mathrm{V}$ and an objective function $f(\mathbf{x})$ as described in Equation 13:

$$
f(x, y)=-\sqrt{x^{2}+y^{2}}
$$

The function was normalised such that $f(\mathbf{x}) \in[0,1]: \forall x \in$ $\left[\mathbf{x}_{\text {min }}, \mathbf{x}_{\text {max }}\right]$. Fig. 2 shows the heatmap and surface plot. The function represents a simple problem with a clear gradient.

TABLE V. Constants SetTings for The Model

\begin{tabular}{|c|c|c|c|c|c|c|}
\hline Constants & $T_{\max }$ & $N_{p o p}$ & $\mathbf{V}_{\max }$ & $\mathbf{x}_{\min }$ & $\mathbf{x}_{\max }$ & $\mathbf{V}_{\text {init }}$ \\
\hline Values & 25 & 4 & 5.0 & $(-100,-100)$ & $(100,100)$ & $(1.0,1.0)$ \\
\hline
\end{tabular}



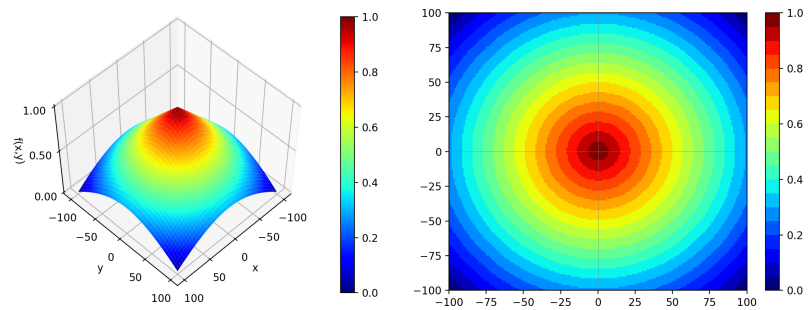

Fig. 2. Surface plot (left) and heatmap (right) for normalised Equation 13 with a maximum in $(0,0)$. Colour ranges from blue (minimum) to red (maximum).

\section{Using a Genetic Algorithm to Evolve Starting POSITIONS}

In this work we investigate whether teams of individuals who share similar initial ideas about the task perform better at solving the task, compared to teams who begin with very different initial ideas. In the model an "initial idea" corresponds to the starting point of the agent in the solution space. Thus, we use a genetic algorithm to evolve the starting points of agents, and then run the model to determine their success (team fitness).

The starting position of each team member is a point on a circle with origin at the optimal so that no starting position is more advantageous than another. Positions are represented using polar coordinates, $\mathrm{P} n(r, \theta)$ where radial $r=90$ (i.e., a distance of 90 from $(0,0)$ ), and polar coordinate angle $\theta$ determined by the GA. Each $\theta$ is represented as a 9-digit binary chromosome. This string is converted into degrees by converting the binary string to its decimal equivalent, which ranges from 0 to 511, adding 1 to it to make it range from 1 to 512 , and then scaling it to 360 degrees. For a team of $n=4$ members as used in the experiments, their angles are specified by a 36-digit binary genotype. For example, the 36-digit genotype 011001000010001100101011111000110001 corresponds to the phenotype illustrated in Fig. 3.

\begin{tabular}{|c|c|}
\hline $\mathbf{P} \mathbf{1}_{\boldsymbol{\theta}}$ & $\mathbf{P 2 _ { \boldsymbol { \theta } }}$ \\
\hline $011001000=200$ & $010001100=140$ \\
\hline$\frac{200+1}{512} \times 360^{\circ}$ & $\frac{140+1}{512} \times 360^{\circ}$ \\
$=141^{\circ}$ & $99^{\circ}$ \\
\hline $\mathbf{P 3}_{\boldsymbol{\theta}}$ & $\mathbf{P 4}_{\boldsymbol{\theta}}$ \\
\hline $10101111=351$ & $000110001=49$ \\
\hline$\frac{351+1}{512} \times 360^{\circ}$ & $\frac{49+1}{512} \times 360^{\circ}$ \\
$=248^{\circ}$ & $=35^{\circ}$ \\
\hline
\end{tabular}

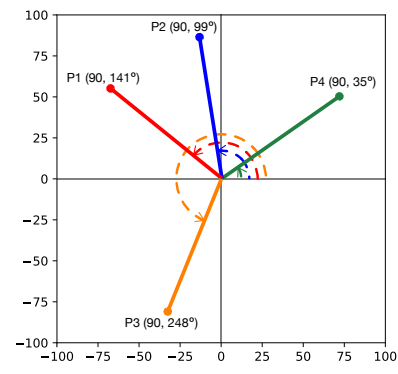

Fig. 3. Positions of team members P1, P2, P3 and P4 in problem space D.

A standard canonical GA is used with population size of 20 , each individual solution representing the starting positions of a team. Each team has four members with predefined personalities (one of the 16 types). For each member of the population, fitness is calculated by decoding the genotype to produce four starting locations for the team members. The agent-based model is run 50 times for the team to produce average team performance for the task of maximising Equation 13. (The group best at the end of each run is recorded and team performance is measured by their average group best, which is the total group best for all runs divided by total number of runs). Based on fitness, 8 individuals are chosen as parents. 20 child teams are created using singlepoint crossover from the parents. A single bit flip mutation occurs with a probability of 0.2 per chromosome. The GA is run for 100 generations. (Values were found following preliminary experiments to determine fastest and most effective settings.)

We can then understand the diversity of "initial ideas" as the average starting distance in polar coordinate angles between team members:

$$
\operatorname{StartDist}_{1,2,3,4}=\frac{D_{1,2}+D_{1,3}+D_{1,4}+D_{2,3}+D_{2,4}+D_{3,4}}{6}
$$

where we calculate the nearest distance between two members $P i$ and $P j$ as $D_{i, j}=\left|P i_{\theta}-P j_{\theta}\right|$ if $\left|P i_{\theta}-P j_{\theta}\right| \leq 180^{\circ}$ and $D_{i, j}=360^{\circ}-\left(\left|P i_{\theta}-P j_{\theta}\right|\right)$ if $\left|P i_{\theta}-P j_{\theta}\right|>180^{\circ}$. In a team of four, the highest possible StartDist is $120^{\circ}$ and occurs when each consecutive team member is $90^{\circ}$ apart.

\section{EXPERIMENTS}

To investigate the effects of differing initial ideas within teams, we use the GA to set the "initial idea" of each member of the team. Our experiments investigate four scenarios:

E1. What are the optimal initial positions of team members, in order for the team to achieve best success in solving their problem, where all individuals share the same personalities?

E2. What are the optimal initial positions of team members, in order for the team to achieve worst success in solving their problem, where all individuals share the same personalities?

E3. What are the optimal initial positions of team members, in order for the team to achieve best success in solving their problem, where all individuals have random personalities?

E4. What are the optimal initial positions of team members, in order for the team to achieve worst success in solving their problem, where all individuals have random personalities?

For E1 and E2, to investigate starting positions where all team members have the same personalities, we repeated the experiment for 16 teams, one for each personality type, with all four team members having the same personality (e.g., a team of four INTJs). E1 used parent selection of the best members of the population, with the best team being the top team from the final generation. E2 used parent selection of the worst members of the population with the worst team being the bottom team from the final generation.

For E3 and E4, the number of possible teams with random personalities can be calculated as:

$$
C^{R}(n, r)=\frac{(n+r-1)}{r !(n-1) !}, C^{R}(16,4)=3876
$$

Each GA takes an average of 2 hours per run, depending on the personality combinations, so investigating all combinations of teams is infeasible. For this reason, we randomly selected 20 teams of random personality combinations, shown in Table VI. The same GA parent and final team selection was used in E3 and $\mathrm{E} 4$ as for E1 and E2, respectively. 
TABLE VI. TEAMS WITH RANDOM PERSONALITIES

\begin{tabular}{|c|c|c|c|c|}
\hline Team No. & P1 & P2 & P3 & P4 \\
\hline T1 & INTJ & ISTP & ESFP & ENFP \\
\hline T2 & INFJ & INFJ & INTJ & INTP \\
\hline T3 & INFJ & ENTP & ENTP & ENTJ \\
\hline T4 & INTJ & INTP & INTP & ENFJ \\
\hline T5 & ISTJ & ISTJ & ISFP & ESFJ \\
\hline T6 & INFJ & ISFP & ENFJ & ENTJ \\
\hline T7 & ISFJ & INFP & ESTJ & ESFJ \\
\hline T8 & ISFJ & INTJ & ENFP & ENTP \\
\hline T9 & ESTP & ENFP & ESFJ & ENTJ \\
\hline T10 & ISTJ & ISFJ & INTP & INTP \\
\hline T11 & INFJ & INFJ & ISTP & ENFP \\
\hline T12 & ISFJ & INFP & ESTP & ENFP \\
\hline T13 & ISFJ & INFP & INFP & ESTJ \\
\hline T14 & ISFJ & ESFP & ESFP & ENTP \\
\hline T15 & INTJ & INTJ & ISFP & ESFP \\
\hline T16 & ISTJ & ISTJ & INTP & ESTP \\
\hline T17 & INFJ & ISTP & ISFP & ENFJ \\
\hline T18 & INFJ & ESTP & ENFJ & ENTJ \\
\hline T19 & INTP & ESTP & ENFP & ESFJ \\
\hline T20 & ISFJ & INFJ & ISFP & ENFP \\
\hline
\end{tabular}

For all experiments, we ran the GA 10 times and measured average StartDist over 10 runs (AvgDist), average team performance over 10 runs (AvgPerf), and finally, $t$-test is used to assess whether the differences in the averages between the best teams and their corresponding worst teams are significant.

\section{RESULTS}

\section{A. Experiments E1 and E2: Teams with Same Personality}

Fig. 4 provides the results for experiments E1 and E2. Most best teams (E1) have more diverse initial ideas compared to worst teams (E2) - 14 out of 16 have higher AvgDist, with Teams INTJ and ENFJ being the exceptions (Fig. 4 top). All best teams (E1) perform significantly better than their corresponding worst teams (E2) (Fig. 4 bottom). The differences in AvgDist between E1 and E2 for Teams ISTJ, INFJ, INTJ, INTP, ENFP, and ENFJ are not significant, indicating that they are less sensitive, with similar distances between best and worst teams.

When optimised, Team INFJ performed the best (0.9999, AvgDist $\left.=105.6^{\circ}\right)$ and INTP the worst $\left(0.9552\right.$, AvgDist $\left.=95.2^{\circ}\right)$. When minimised, Team INFJ performed the best $(0.9878$, AvgDist $\left.=99.9^{\circ}\right)$ and ISFP the worst $\left(0.5795\right.$, AvgDist $\left.=37.3^{\circ}\right)$. The GA found team ISTP to be the most sensitive to distance between team members with the highest $A v g D i s t$ of $116.8^{\circ}$ (very near the maximum of $120^{\circ}$ ) when optimised and the lowest AvgDist of $21.4^{\circ}$ when minimised.

\section{B. Experiments E3 and E4: Teams with Random Personality}

Fig. 5 provides the results for experiments E3 and E4. Random teams are less sensitive to diversity of initial position amongst team members. Although most best teams (E3) have more diverse initial ideas compared to worst teams (E4) (12 out of 20 have higher $A v g D i s t$ ) the differences are only significant for six of the teams (Fig. 5 top). None of the teams with INFJ and ENFJ members have significant differences in AvgDist. This appears to mirror findings for E1 and E2, where INFJ and ENFJ are among the personalities whose performance are not sensitive to diversity. All best teams (E3) perform significantly better than their corresponding worst teams (E4) (Fig. 5 bottom).

Team T4 is the only team where when optimised (E3) has significantly lower AvgDist than when minimised (E4). T4 consists fully of team members that are not sensitive to initial positions (as found in E1 vs. E2). The results also show that interaction between personalities changes their sensitivity to diversity. For example, everyone in T14 is sensitive to distances in E1 vs. E2, but the differences in AvgDist for E3 vs. R4 are not significant; T10 and T16 has 3 out of 4 team members that are not sensitive to distances in E1 vs. E2 but both have high significant difference in AvgDist when put with one member where distances matter.

\section{ANALYSIS}

Fig. 6 illustrates the change in AvgDist for each team in each experiment during evolution. Most teams have their AvgDist increased over generations for E1 and E3, and decreased over generations for E2 and E4. However, it is clear that the GA discovered that making distances as large as possible, or as small as possible, was rarely optimal. Instead, the GA converged to relatively stable specific distance values for each team. E1 and E2 show that most personalities are more effective with larger initial distances, with only two exceptions: INTJ and ENFJ, which seem to work well regardless of distance. A similar result is clear in E3 and E4, where the right combination of different personalities can perform consistently well, making the team less affected by initial distances. Thus, in general, diversity is helpful, whether in initial position or in team personalities.

Fig. 7 illustrates the change in AvgPerf for each team in each experiment during evolution. All teams are able to optimise their AvgPerf in E1 and E3 and minimise it in E2 and E4, although it

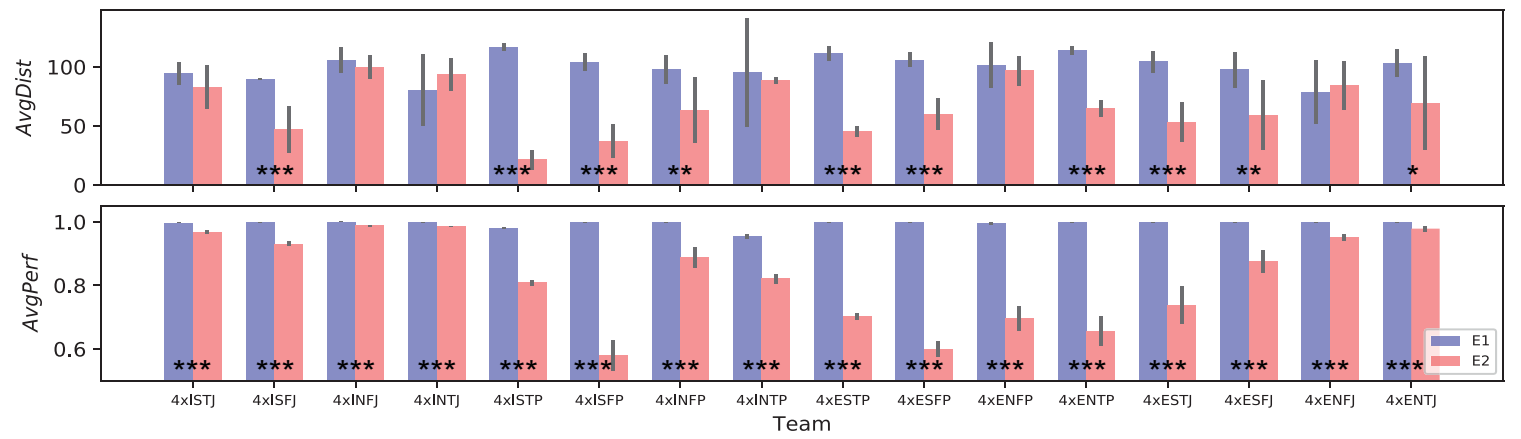

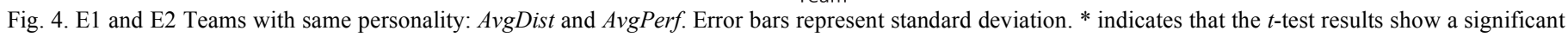
difference between the averages for the pair of teams at $p<.05$, ** at $p<.01$ and $* * *$ at $p<.001$. 

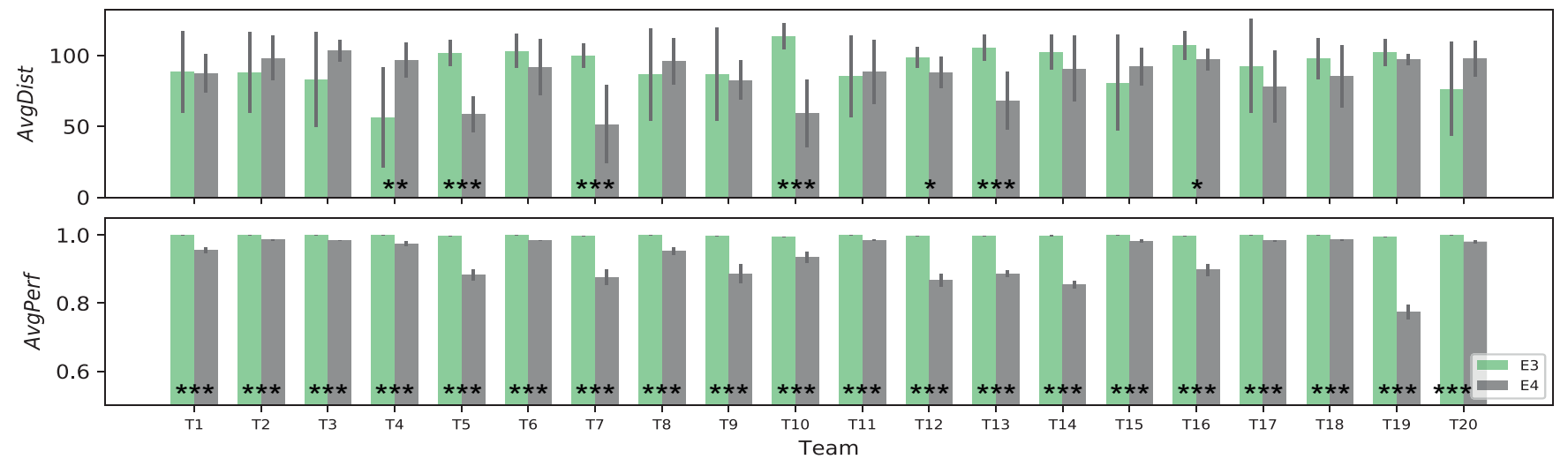

Fig. 5. E3 and E4 Teams with random personality: AvgDist and AvgPerf. Error bars represent standard deviation. * indicates that the $t$-test results show a significant difference between the averages for the pair of teams at $p<.05, * *$ at $p<.01$ and $* * *$ at $p<.001$.
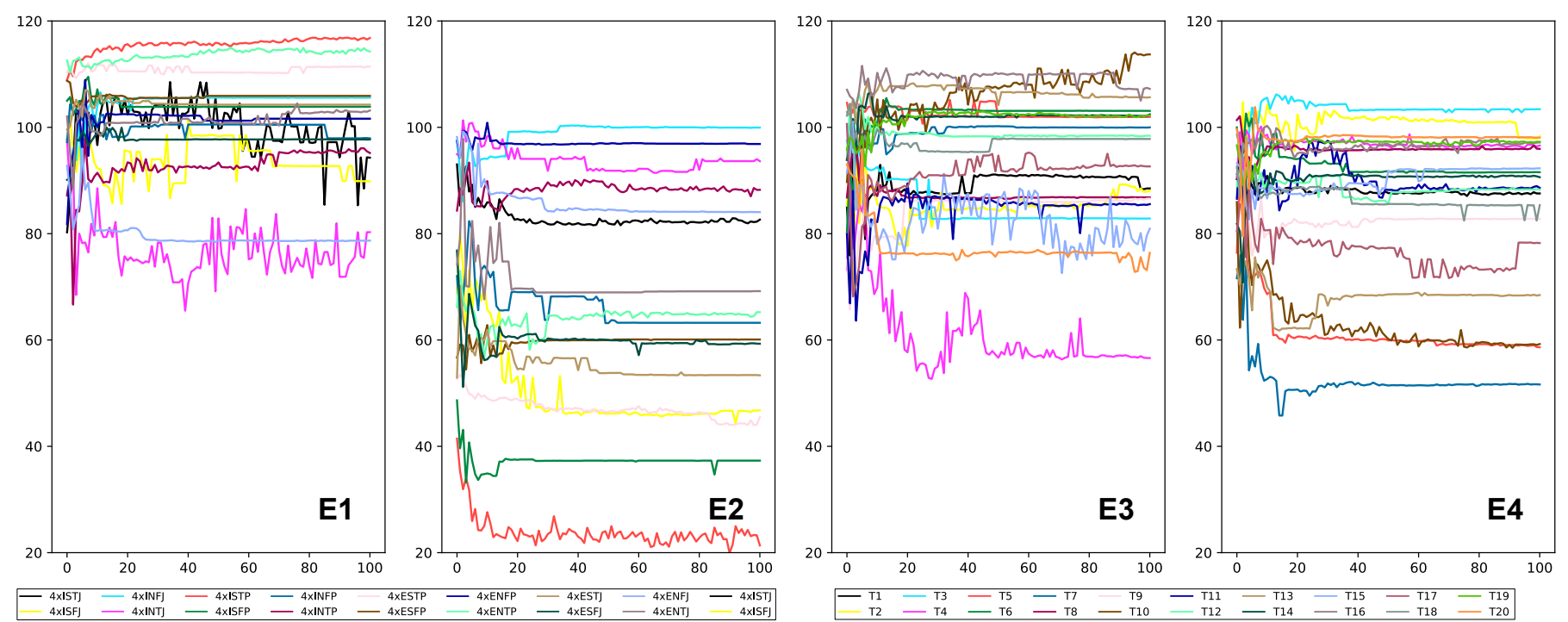

Fig. 6. AvgDist ( $y$-axis) over generations ( $x$-axis) for E1, E2, E3 and E4. All experiments share the same $y$-axis scales.
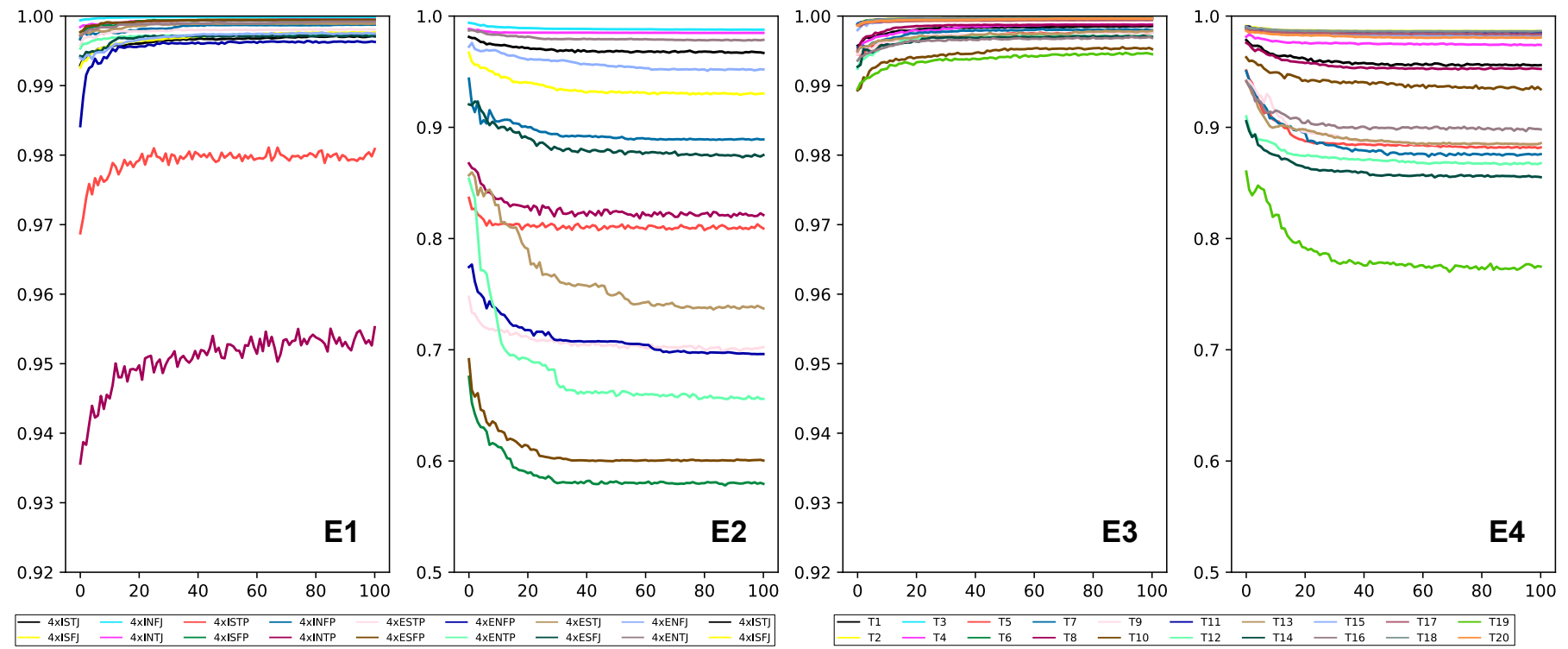

Fig. 7. AvgPerf (y-axis) over generations ( $x$-axis) for E1, E2, E3 and E4. E1 and E3 share the same $y$-axis scales. E2 and E4 share the same $y$-axis scales. 
is clear that some teams produce considerably better results when minimising their performance, compared to others.

Some explanation for these behaviours can be found by looking at the significances of Fig. 4 and Fig. 5, where the Se function (Table IV) is present in all teams that have significant differences in AvgDist between E1 and E2 (but not between E3 and E4), while the Ni function (Table IV) is the most indifferent to distances, i.e., the majority of homogeneous teams with $\mathrm{Ni}$ are indifferent to distances, and uniquely all heterogeneous teams with the Ni function are indifferent to distances. Thus, the model predicts that Extraverted Sensors (who rely on communication) do better with teams containing diverse ideas, while Introverted Intuitives (who are inward thinking and rely less on communication) are less affected by diversity.

Looking at average distances and performances across all experiments (Fig. 8), best teams have higher average AvgDist compared to their corresponding worst teams, i.e., E1 vs. E2 and E3 vs. E4. This confirms that regardless of personality (same or random), teams with higher diversity in their initial positions perform better than teams with lower diversity. The average AvgDist of best teams with same personalities (E1) is higher than the average AvgDist of best teams with random personalities (E3), indicating that when team members have same personalities, they needed to start from more diverse ideas in order to perform their best. This prediction is corroborated by literature: empirical studies have found that functionally diverse management teams are more innovative, respond better to threats, and quicker to implement organisational change than functionally homogeneous teams. In a study involving 92 workgroups, informational diversity, i.e., differences in knowledge and perspectives that members bring to the team, positively influences team performance [15].

Teams with different personalities are more resilient to the differences in initial positions affecting performances. As can be seen in Fig. 8 (right), heterogeneous teams perform better than homogeneous teams with smaller standard deviations, regardless of whether it is minimisation or maximisation. This prediction is also corroborated by literature: separate studies of information systems teams have found that successful teams have more diverse personalities [16, 17].
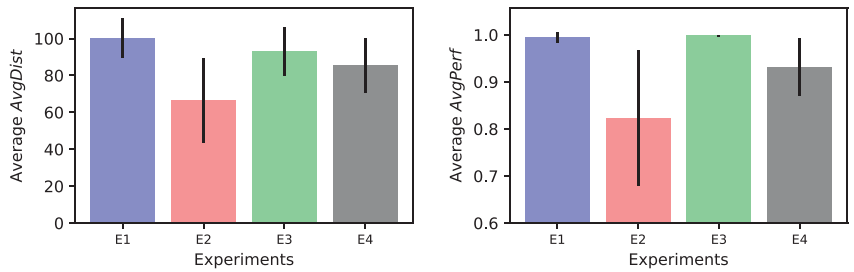

Fig. 8. Average AvgDist (left) and average AvgPerf (right) over all teams in E1, E2, E3 and E4. Error bars represent standard deviation.

\section{CONCLUSION}

In this work we applied a genetic algorithm to an agentbased model of collaboration in order to explore the effects of creating teams with diverse or similar initial ideas on their ability to solve a simple task. As discovered by the GA, each type of team "preferred" a different and very specific level of diversity for them to work optimally well or poorly. The results showed that almost all homogeneous teams (comprising identical personality types) benefited from starting from more diverse initial ideas (and those that did not benefit were already performing well so no benefit was evident). At their worst, homogeneous teams were also considerably worse than heterogenous teams. The results also showed that heterogeneous teams (comprising mixtures of personalities) generally performed the task more consistently well, and while many also benefited from specific levels of diversity in their initial positions, some were naturally diverse enough that it made little difference to their already-good results.

In optimisation and machine learning, the benefits of broader exploration before fine-tuning via exploitation are well-known. It seems that the same may also be true of human teams: the right amount of diversity can enable improved exploration of the problem, and better, more consistent results.

\section{REFERENCES}

[1] S. L. Lim and P. J. Bentley, "Coping with uncertainty: modelling personality when collaborating on noisy problems," in The 2018 Conference on Artificial Life (ALIFE), 2018, pp. 566-573.

[2] J. Salvit and E. Sklar, "Modulating agent behavior using human personality type," in Workshop on Human-Agent Interaction Design and Models (HAIDM), 2012, pp. 145-160.

[3] C. Jung, Psychological Types. Oxford, England: Harcourt, Brace, 1923.

[4] A. Campos, F. Dignum, V. Dignum, A. Signoretti, A. Magály, and S. Fialho, "A process-oriented approach to model agent personality," in International Conference on Autonomous Agents and Multiagent Systems (AAMAS), vol. 2, 2009, pp. 1141-1142.

[5] S. Ahrndt, J. Fähndrich, and S. Albayrak, "Modelling of personality in agents: from psychology to implementation," in Workshop on HumanAgent Interaction Design and Models (HAIDM), 2015, pp. 1-16.

[6] L. R. Goldberg, "An alternative "description of personality": the big-five factor structure," Journal of Personality and Social Psychology, vol. 59, p. 1216, 1990.

[7] F. Durupinar, N. Pelechano, J. Allbeck, U. Gudukbay, and N. I. Badler, "How the ocean personality model affects the perception of crowds," IEEE Computer Graphics and Applications, vol. 31, pp. 22-31, 2011.

[8] D. B. Knoester, H. J. Goldsby, and P. K. McKinley, "Genetic variation and the evolution of consensus in digital organisms," IEEE Transactions on Evolutionary Computation, vol. 17, pp. 403-417, 2013.

[9] R. E. Lenski, C. Ofria, R. T. Pennock, and C. Adami, "The evolutionary origin of complex features," Nature, vol. 423, p. 139, 2003.

[10] A. J. Heppenstall, A. J. Evans, and M. H. Birkin, "Genetic algorithm optimisation of an agent-based model for simulating a retail market," Environment and Planning B: Planning and Design, vol. 34, pp. 1051$1070,2007$.

[11] Z. J. Wang, X. F. Xu, and D. C. Zhan, "Genetic algorithm for collaboration cost optimization-oriented partner selection in virtual enterprises," International Journal of Production Research, vol. 47, pp. 859-881, 2009.

[12] S. Ahrndt, A. Aria, J. Fähndrich, and S. Albayrak, "Ants in the OCEAN: Modulating agents with personality for planning with humans," in European Conference on Multi-Agent Systems (EUMAS), 2014, pp. 3-18.

[13] I. B. Myers, The Myers-Briggs Type Indicator: Manual. Palo Alto, California: Consulting Psychologists Press, 1962.

[14] C. K. Williams and C. E. Rasmussen, "Gaussian processes for regression," in Advances in Neural Information Processing Systems (NIPS), 1996, pp. 514-520.

[15] K. A. Jehn, G. B. Northcraft, and M. A. Neale, "Why differences make a difference: a field study of diversity, conflict and performance in workgroups," Administrative Science Quarterly, vol. 44, pp. 741-763, 1999.

[16] K. B. White, "MIS project teams: an investigation of cognitive style implications," MIS Quarterly, pp. 95-101, 1984.

[17] J. H. Bradley and F. J. Hebert, "The effect of personality type on team performance," Journal of Management Development, vol. 16, pp. 337$353,1997$. 